\title{
Scalp Melanoma Treated with Ipilimumab in Elderly Patient: A Complete Response to Treatment-Case Report
}

Juliana Ramos Chaves ${ }^{1}$, Filipe Rocha da Silva ${ }^{1,2}$, Lucas Ivanilson Guedes Henriques Silva ${ }^{1,3}$, Thiago Farias Câmara $^{1,2}$, Luis Eduardo Werneck de Carvalho ${ }^{1}$ and Jonathan Souza Sarraf ${ }^{1,4}$

1. Oncologica Brasil-Education and Research, Belém-PA 66053-000, Brazil

2. Mecicine Faculty, Universidade do Estado do Pará, Belém-PA 66087-670, Brazil

3. Medicine Faculty, Centro Universitário do Estado do Pará, Belém-PA 66613-903, Brazil

4. Medicine Faculty, Universidade Federal do Pará, Belém-PA 66075-110, Brazil

\begin{abstract}
Melanoma is a malignant neoplasm that affects the melanocytes. Its treatment is based on cancer staging. In immunotherapy, it can be pointed out the ipilimumab, used in the systemic therapy for unresectable and/or metastatic melanoma in the first or the second line treatment with increased survival. However, limiting toxicity and the low complete response discourage its use in the elderly patients. The aim of this study is to report the case of an 83-year-old man, white, with scalp melanoma measuring $3.5 \mathrm{~cm}$ in its major axis. After two resections was verified that the deep margin was involved, making the lesions unresectable. The evaluation of distant metastasis, by skull, chest and abdomen CT-scan (computed tomography-scan), was performed and did not detect the presence of any metastatic foci. The patient underwent the first cycle of chemotherapy with DTIC (dacarbazine) protocol. After the first cycle, the medication was discontinued due to significant myelotoxicity and Grade 3 thrombocytopenia. Underwent second line treatment with ipilimumab by four cycles. After this, the patient follow with complete local response, asymptomatic, with no signs of local recurrence or distant metastasis site, and no limiting toxicity during and after a year of treatment with ipilimumab. Thus, it is possible to suggest that the use of ipilimumab isolatedly as a second line treatment may have a good response even in elderly patients.
\end{abstract}

Key words: Scalp melanoma, ipilimumab, elderly patients.

\section{Introduction}

Melanoma is a malignant neoplasm that affects the melanocytes. Although it is the most rare skin cancer, with an overall incidence of 15 20 cases per 100,000 people, melanoma causes the majority $(75 \%)$ of skin cancer deaths $[1,2]$.

The treatment of melanoma is based on cancer staging. In unresectable Stage III, Stage IV and in cases of recurrence, the treatment may include chemotherapy, inhibitors of signal transduction and immunotherapy [1, 2]. In immunotherapy, it can be pointed out the ipilimumab, IgG1 monoclonal antibody which plays an important role in the inhibition of the

Corresponding author: Juliana Ramos Chaves, M.D., research field: oncology.
CTLA-4 antigen by stimulating the activation and proliferation of anti-tumor $\mathrm{T}$ cells [3-5].

Ipilimumab is used in the systemic therapy for unresectable and/or metastatic melanoma [6-8] in the first [7] or the second line [6] treatment with increased survival. However, toxicity rates $[6,7]$ and the low complete response rates $[6,7,9]$ discourage its use in the elderly patients.

Thus, the aim of this study is to report the case of an elderly patient with scalp melanoma, with complete local response and no limiting toxicity during and after a year of treatment with ipilimumab.

\section{Case Presentation}

The 83-year-old man, white, assisted in October 
2013 at the D'or Oncology service in Rio de Janeiro, Brazil. The patient had a tumor in the scalp measuring $3.5 \mathrm{~cm}$ in its major axis. After biopsy followed by pathology and immunohistochemistry tests, it was diagnosed as poorly differentiated malignant neoplasm with CD68 markers, positive Melan A antigen and vimentin (melanoma).

After diagnosis, the patient underwent two surgical procedures in an attempt to perform complete tumor resection, but without success and with a gradual increase of the injury. In March 2014, a new surgical resection was performed with removal of $7 \mathrm{~cm}$ of the lesion, but it was verified that the deep margin was involved, making the lesions unresectable. The evaluation of distant metastasis, by skull, chest and abdomen CT-scan (computed tomography-scan), was performed in February 2014 and did not detect the presence of any metastatic foci.

In May 2014, the patient underwent the first cycle of chemotherapy with DTIC (dacarbazine) protocol $1,000 \mathrm{mg} / \mathrm{m}^{2}$. After the first cycle, the medication was discontinued due to significant myelotoxicity and Grade 3 thrombocytopenia (Platelet $=30,000)$. Thus, there was disease progression and treatment change.

In July 2014, the patient underwent second line treatment with ipilimumab $(3 \mathrm{mg} / \mathrm{kg}$ every 21 days for four cycles) [7]. The lesion at the beginning of treatment with ipilimumab is shown in Fig. 1. After two cycles of ipilimumab, the patient had partial clinical response, evaluated by Fig. 2 and performance status. After four cycles, the clinic response was complete (Fig. 3).

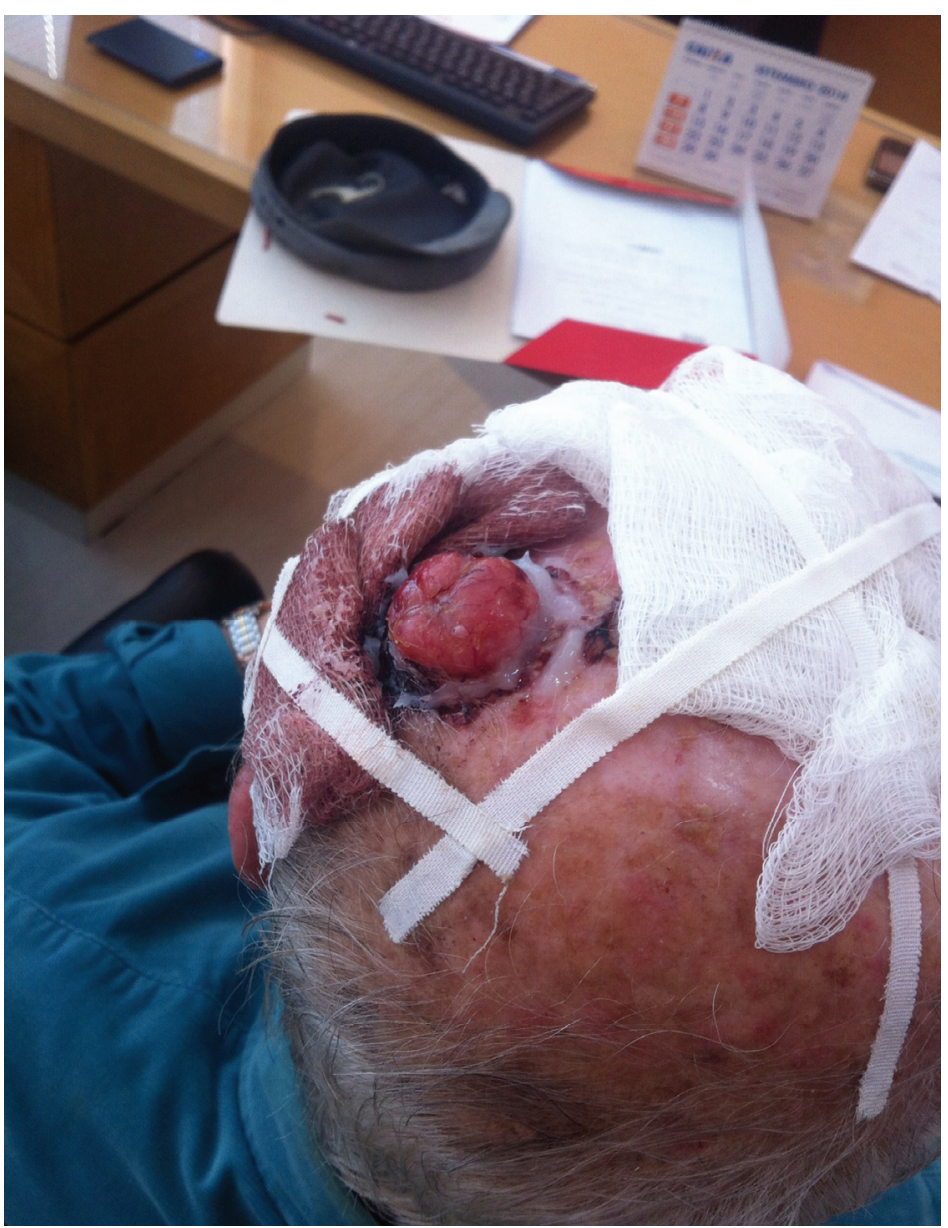

Fig. 1 Lesion at the beginning of treatment. 


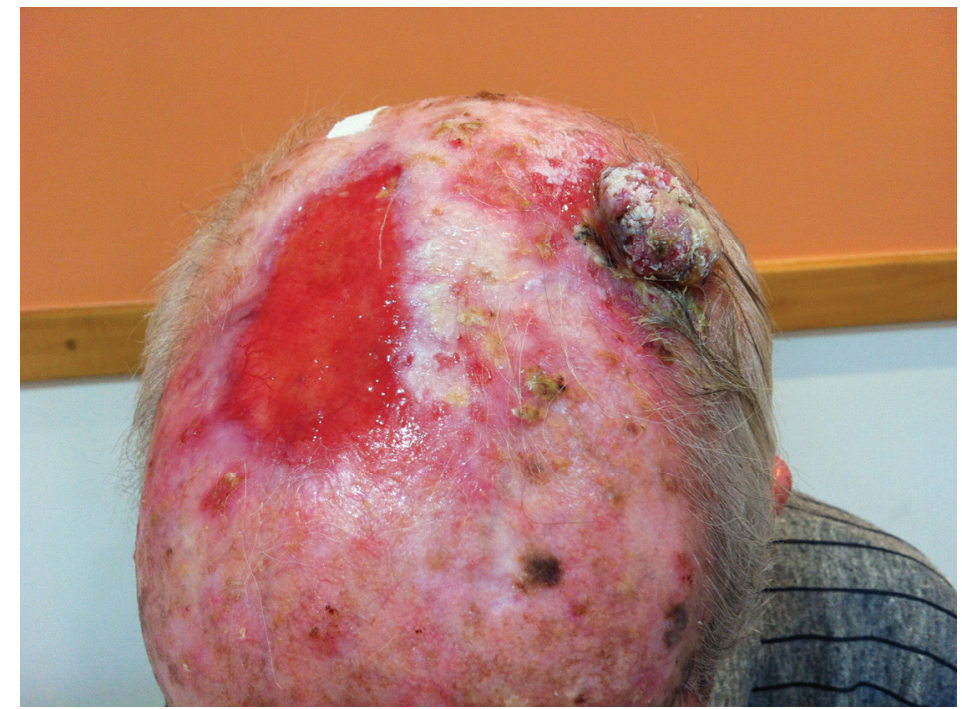

Fig. 2 Lesion after two cycles of ipilimumab.

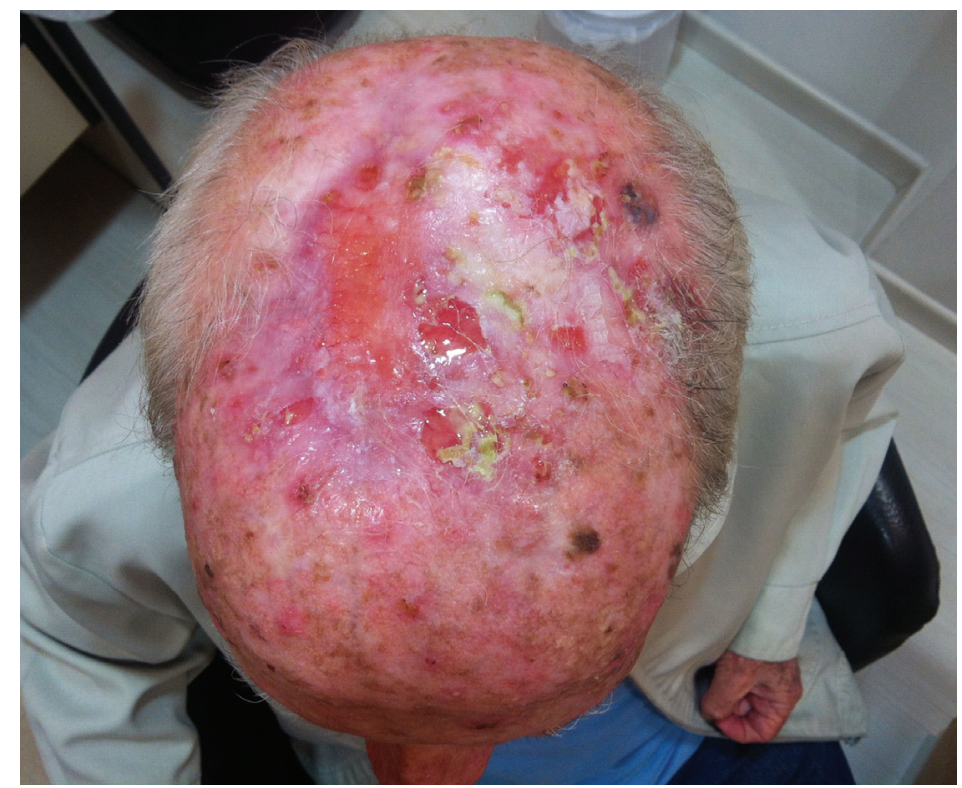

Fig. 3 The result after four cycles of ipilimumab.

In October 2014, the cycles were discontinued without toxicity related to the treatment. One year after completion of treatment with ipilimumab and 24 months after diagnosis, the patient is asymptomatic, with no signs of local recurrence or distant metastasis site, and does not show evidence of late toxicity. The patient is in bi-monthly follow-up by the medical oncology service.

\section{Discussion and Conclusions}

In this case, the 83-year-old patient with Stage III melanoma (T4b, NX, M0) showed complete response after four cycles of ipilimumab as second line treatment, after attempts to resection and therapy with DTIC as first line treatment, which presented myelotoxicity.

Comparing to our patient ( 83 years old), it is noteworthy that in Phase III studies, the average age of the patients treated with ipilimumab isolatedly was 56.8 years [6] and the average age of those treated with ipilimumab + dacarbazine was 57.5 years [7]. In addition, individuals younger than 65 years showed a better response to the treatment with ipilimumab + 
dacarbazine [7], compared to older individuals. In the Italian expanded access program, 188 patients older than 70 years ( 27 older than 80 years) and with immune control of the disease were evaluated. Regarding the efficacy and safety of treatment with ipilimumab, there was no difference compared to younger patients [9].

Clinical trials show that the melanoma complete response was achieved in around $1.5 \%$ of subjects in both treatments using ipilimumab isolatedly as second line treatment [6], and in ipilimumab + dacarbazine as first line treatment [7]. Furthermore, a study evaluating the efficacy of treatment according to age showed that only $12.2 \%$ of patients had complete response [9]. Despite the low levels of complete response, our patient presents this same response with four cycles of ipilimumab as second line monotherapy, thus showing a good response to this drug.

The use of ipilimumab monotherapy as second-line treatment provided the patients with an average survival of 10.1 months, compared to the use of Gp100 monotherapy which showed an average survival of 6.4 months [6]. Also, another Phase III study, which presents the ipilimumab associated with dacarbazine in the first line, compared with the isolated use of them, showed an increase of 2.1 months in the survival the subjects [7]. These data, on the use of ipilimumab in the treatment of melanomas, are clear in our report, given that our patient is with 24 months of survival after diagnosis and 12 months of disease-free survival after completion of therapy with ipilimumab isolatedly.

The main toxicities during the use of ipilimumab are autoimmune skin events (rash and itching) [10], gastrointestinal events (diarrhea) [6, 7], and long-term reactions, such as vitiligo, hypopituitarism and cholelithiasis [6]. Nevertheless, the patient in our study showed no adverse reactions during treatment and no signs of autoimmune reactions later.

The data presented show complete response to the use of ipilimumab in elderly patient with unresectable Stage III melanoma. In addition, no toxicity was observed in short and long term. Thus, it is possible to suggest that the use of ipilimumab isolatedly as a second line treatment may have a good response even in elderly patients.

\section{References}

[1] Schadendorf, D., Fisher, D. E., Garbe, C., Gershenwald, J. E., Grob, J. J., Halpern, A., et al. 2015. "Melanoma." Nat. Rev. Dis. Prim. 1: 15003.

[2] American Cancer Society. 2015. "Melanoma Skin Cancer." Accessed March 15, 2016. American Cancer Society. http://www.cancer.org/cancer/skincancer-melanoma/detai ledguide/melanoma-skin-cancer-what-is-melanoma.

[3] Snyder, A., Makarov, V., Merghoub, T., Yuan, J., Zaretsky, J. M., Desrichard, A., et al. 2014. "Genetic Basis for Clinical Response to CTLA-4 Blockade in Melanoma." N. Engl. J. Med. 371: 2189-99.

[4] Weber, J. S., O’Day, S., Urba, W., Powderly, J., Nichol, G., Yellin, M., et al. 2008. "Phase I/II Study of Ipilimumab for Patients with Metastatic Melanoma." J. Clin. Oncol. 26: 5950-6.

[5] Maio, M., Grob, J. J., Aamdal, S., Bondarenko, I., Robert, C., Thomas, L., et al. 2015. "Five-Year Survival Rates for Treatment-Naive Patients with Advanced Melanoma Who Received Ipilimumab Plus Dacarbazine in a Phase III Trial." J Clin Oncol. 33: 1191-6.

[6] Hodi, F. S., O’Day, S. J., McDermott, D. F., Weber, R. W., Sosman, J. A., Haanen, J. B., et al. 2010. "Improved Survival with Ipilimumab in Patients with Metastatic Melanoma." N. Engl. J. Med. 363: 711-23.

[7] Robert, C., Thomas, L., Bondarenko, I., O’Day, S., Weber, J., Garbe, C., et al. 2011. "Ipilimumab Plus Dacarbazine for Previously Untreated Metastatic Melanoma." N. Engl. J. Med. 346: 2517-26.

[8] Du Rusquec, P., Saint-Jean, M., Brocard, A., Peuvrel, L., Khammari, A., Quéreux, G., et al. 2014. "Ipilimumab-Induced Autoimmune Pancytopenia in a Case of Metastatic Melanoma." J. Immunother. 37: 348-50.

[9] Chiarion Sileni, V., Pigozzo, J., Ascierto, P. A., Grimaldi, A. M., Maio, M., Di Guardo, L., et al. 2014. "Efficacy and Safety of Ipilimumab in Elderly Patients with Pretreated Advanced Melanoma Treated at Italian Centres through the Expanded Access Programme.” J. Exp. Clin. Cancer Res. 33: 30.

[10] Ludlow, S. P., and Kay, N. 2015. "Delayed Dermatologic Hypersensitivity Reaction Secondary to Ipilimumab.” $J$. Immunother. 38: 165-6. 